Artículo

\title{
Moodle como herramienta de evaluación: la experiencia del Bachillerato de Informática Empresarial en la UCR Sede de Occidente
}

Moodle as an assessment tool: the experience of the Bachelor of Business Computing career at the western campus of Universidad de Costa Rica

Sindy Porras Santamaría*

sindy.porras@ucr.ac.cr

ISSN 1996-1642, Editorial Universidad Don Bosco, año 10, No.17, enero-junio de 2016, pp. 53-60 Recibido: 14 de mayo 2015. Aprobado: 29 de noviembre 2015.

Resumen

El presente artículo describe la experiencia personal en el uso de Moodle en la carrera de Bachillerato en Informática Empresarial en la Sede de Occidente de la Universidad de Costa Rica. Se destaca aquí las facilidades que proporciona como una propuesta de evaluación emergente a través de diferentes funcionalidades tanto para la interacción con los estudiantes como las alternativas que ofrece a los procesos tradicionales de evaluación. Se busca además compartir los resultados y logros obtenidos con su uso a lo largo de los años en los diferentes cursos de la carrera.

Palabras clave: Moodle, evaluación emergente, Universidad de Costa Rica: Informática Empresarial.
Abstract:

This paper overviews the personal experience on the use of Moodle and its assessment tools at the Business Computing Bachelor career at the Western Campus of University of Costa Rica. It brings out the possibilities as an emerging evaluation tool by means of a variety of applications and devices for both student interaction and assessment as alternate choices to traditional evaluation processes. In this paper, the author also shares the experience outcomes gathered through years of virtual teaching.

Keywords: Moodle, emergent evaluation, University of Costa Rica, Enterprise Computing. 


\section{Introducción}

A lo largo de los años los procesos de evaluación de aprendizajes han ido evolucionando de acuerdo con los nuevos paradigmas educativos, los cambios sociales y a la evolución tecnológica. Ahora, sin embargo, se siguen utilizando muchas de las alternativas tradicionales de evaluación, como la prueba escrita y la prueba oral a pesar de las nuevas opciones que han emergido.

En el nivel universitario se han desarrollado las modalidades de cursos bimodales y cursos virtuales en diferentes instituciones educativas alrededor del mundo. En la Universidad de Costa Rica (UCR) se ha implementado la opción de cursos bimodales y se está trabajando en el desarrollo de nuevas opciones académicas totalmente enfocadas a la virtualidad, mediante el 100\% de sus cursos en línea. Una de las herramientas que ofrece la UCR para que los docentes la utilicen es la Mediación Virtual. Esta herramienta, basada en la plataforma Moodle tiene como finalidad colaborar con sus usuarios en el desarrollo de clases en línea, o como apoyo a las lecciones presenciales.

La carrera de Bachillerato en Informática Empresarial de la Sede de Occidente comenzó a utilizar Mediación Virtual en algunos de sus cursos desde el año 2007 como una opción para mantener un repositorio de materiales de clase, facilitar la entrega de tareas en formato digital y como un medio de comunicación con los estudiantes. La herramienta se siguió utilizando hasta el año 2012, implementando con el paso del tiempo algunas otras funcionalidades como foros de discusión.

A partir del año 2013 se implementó el uso de una plataforma propia de la carrera basada en la versión 2.0 de Moodle la cual fue configurada según las necesidades específicas de los docentes y las características de los cursos, y administrada por un grupo de profesores.

Desde ese entonces, el uso de esta herramienta forma parte del quehacer de los docentes y estudiantes de la carrera y se ha utilizado, entre otras cosas, para el proceso de evaluación de aprendizajes como una opción a los procesos tradicionales.

Este artículo se basa en la experiencia docente personal en la carrera de Informática Empresarial y el uso de las aulas virtuales, así como en la revisión de los procesos de evaluación de aprendizajes utilizando el portal moodle. ucrso.info. Se busca con esto compartir las vivencias desde una perspectiva de procesos de evaluación emergentes que le brinden al lector mayor información sobre las opciones que ofrece la herramienta. Se explican los diferentes tipos de evaluaciones que se pueden implementar con Moodle, y las facilidades para revisar y calificar el trabajo realizado por cada estudiante.

Moodle como herramienta de evaluación: la experiencia del Bachillerato de Informática Empresarial en la UCR Sede de 54. Occidente

\section{Moodle y sus herramientas de evaluación}

El aprendizaje en línea es una idea que nació tiempo atrás, luego de la aparición del Internet y su uso en la educación. De acuerdo con lo que indica Silvio (2000), 
la idea de una Universidad Virtual que realice sus actividades exclusivamente en el ciberespacio se da a finales de la década de los años ochenta, con iniciativas que fueron bastante ambiciosas. Una de ellas fue la University of the World, promovida por el académico norteamericano James Grier Miller.

La idea de cursos virtuales se ha ido materializando en diferentes instituciones educativas. En la actualidad se encuentran gran variedad de opciones académicas universitarias alrededor del mundo, muchas de ellas en la modalidad virtual. Costa Rica no es la excepción y las universidades están implementándola en cursos y carreras completas.

El uso de plataformas educativas viene a colaborar con los docentes y estudiantes en el proceso de aprendizaje en línea y cursos virtuales. De acuerdo con Ros (2008), Moodle nace como fruto de la Tesis de Martin Dougiamas de la Universidad de Perth, en Australia Occidental en el 2002 quien quería una herramienta que facilitara el constructivismo social y el aprendizaje cooperativo. "Moodle" proviene del acrónimo de Modular Object oriented Dynamic Learning Enviromennt (Entorno Modular de Aprendizaje Dinámico Orientado a Objetos).

En los países desarrollados, las universidades han llegado a crear sitios web con un alto nivel de complejidad e interactividad haciendo uso de la plataforma Moodle. La UCR no se ha quedado atrás, y desde el 2007 ofrece a sus docentes y estudiantes la herramienta denominada Mediación Virtual, que permite crear y desarrollar cursos bimodales y virtuales. Existen, además, otros recursos tecnológicos para la docencia que ofrece la Unidad de Apoyo a la Docencia Mediada con Tecnologías de la Información y la Comunicación (METICS).

Moodle es una herramienta de software libre y gratis que ha extendido su uso a nivel mundial por parte de las instituciones educativas. Docentes del Bachillerato de Informática Empresarial de la Sede de Occidente adoptaron esta herramienta y la adaptaron a las necesidades propias de la carrera, y desde el año 2013 se ha implementado su uso en los cursos con una plataforma similar a Medicación Virtual de la UCR, pero administrada por los mismos docentes, denominada Moodle.ucrso.info.

El uso la plataforma Moodle como complemento de los procesos educativos le permite al profesorado hacer uso de aulas virtuales y poder realizar diferentes tipos de actividades. En la carrera, poco a poco la utilización de las aulas virtuales ha ido incrementando, al punto que actualmente el cien por ciento de los cursos con sigla IF la usan.

Molist (2008) asegura que a Moodle "los docentes lo prefieren porque permite muchas formas de dar clase y porque, al ser código abierto, puede modificarse como les convenga. Lo usan sobre todo como complemento de cursos presenciales y también en educación a distancia" (p.1). 
La plataforma cuenta con varios módulos que le brindan la oportunidad de evaluar los aprendizajes, bajo una serie de opciones que se adaptan a las características de los contenidos y tipo de curso. Sin embargo, aunque se utilizan las aulas virtuales, el uso las opciones de evaluación aún es mínimo.

Moodle ofrece diversas opciones que le permite al docente utilizarla para la evaluación de aprendizajes. Casales, Rojas y Paulí (2008) indican que:

Una de las características más interesantes de Moodle es que brinda herramientas que posibilitan al profesor medir el nivel de asimilación de conocimientos y habilidades del estudiante mediante actividades como los cuestionarios, las tareas, los talleres y los foros. Algunas de estas actividades pueden diseñarse con el fin de que el estudiante pueda autoevaluarse (p.8).

Con base en la revisión y uso de la plataforma en diferentes cursos de la carrera, a continuación se incluye una breve explicación de las opciones de evaluación:

- Consulta: Permite al docente hacer una pregunta especificando las posibles respuestas. Los resultados de la elección puede ser publicados cuando los estudiantes hayan completado la respuesta o después, y se pueden mostrar los nombres de los estudiantes o de forma anónima. Se utiliza para realizar una encuesta rápida que estimule a los alumnos a reflexionar sobre un tema, comprobar rápidamente que los estudiantes han entendido algo concreto, o facilitar la toma de decisiones, por ejemplo, permitiéndoles votar sobre algún aspecto relacionado con el curso.

- Cuestionario: El profesor puede diseñar y plantear cuestionarios con preguntas tipo opción múltiple, falso-verdadero, coincidencia, respuesta corta y respuesta numérica. Esta opción califica automáticamente las respuestas, a excepción de las preguntas de tipo ensayo.

- Encuestas predefinidas: La herramienta proporciona una serie de instrumentos que se han mostrado útiles para evaluar y estimular el aprendizaje en entornos en línea, para recopilar información entre los alumnos, que le ayude al docente conocer mejor su clase así como su propia forma de enseñar. Algo que considero desventaja es que ya tienen las preguntas definidas, por lo que no es posible modificarlas.

- Foro: Le permite al docente y a los participantes tener discusiones asincrónicas, sobre un tema en particular, por un período de tiempo establecido. Se ofrecen diferentes tipos de foros que se pueden adaptar a las necesidades docentes y que buscan la participación activa de los estudiantes. En todos los foros, el docente puede permitir que se adjunten archivos a las aportaciones, que respalden lo comentado.

- Glosario: Los participantes pueden crear y mantener una lista de definiciones, de forma similar a un diccionario. También se puede utilizar esta funcionalidad para recoger y organizar recursos o información a lo largo de un curso. 
- Taller: Permite la recopilación, revisión y evaluación por pares del trabajo de los estudiantes. Da la opción a los estudiantes de enviar cualquier contenido digital o escribir el texto directamente en un campo empleando un editor de texto.

- Tarea: Mediante las tareas el docente puede evaluar el aprendizaje de los alumnos una vez que éstos hayan enviado el documento respectivo a la carpeta correspondiente en Moodle.

- Wiki: En esta actividad los estudiantes pueden añadir y editar una colección de páginas web. Un wiki puede ser colaborativo, donde todos pueden editarlo, o puede ser individual.

- Juegos: Diferentes formatos que el profesor puede utilizar para evaluar los aprendizajes, de una manera diferente, e incluso hasta divertida para los estudiantes. Los juegos con que cuenta son: ahorcado, crucigrama, sopa de letras, millonario, sudoku, imagen oculta y libro con preguntas.

Según Reyes, Cabrera, Estévez, Jiménez y Limaya (2005):

todo tipo de actividad evaluativa utilizada promueve el pensamiento independiente, flexible, profundo y creador, así como una memoria racional y lógica acompañada de una imaginación creadora, al tener que diseñar diferentes cursos y talleres dentro de la modalidad de MOODLE, lo que le permite al profesor evaluar el desempeño de sus estudiantes a partir de los conocimientos recibidos en el curso y retroalimentarse (p.4).

Moodle también cuenta con una herramienta denominada "calificador", que permite incluir y observar cada una de las calificaciones por estudiante, y además realiza el cálculo y muestra la nota total y el promedio del curso.

Es función del docente ajustar cada uno de los rubros a evaluar, asigna puntajes y porcentajes, de acuerdo con la evaluación planteada en la carta al estudiante. Lo ideal es que se prepare el aula virtual con los contenidos del curso y las diferentes estimaciones que realizará a lo largo del mismo, antes de que este inicie. Para ello puede acceder al módulo de calificaciones, que se encuentra dentro del aula virtual. Entre las funcionalidades que se ofrecen en este módulo están: letras, escalas y categorías e ítems.

\section{La experiencia y sus resultados}

Lo aprendido como docente en los cursos Compromiso Social de la Informática y Auditoría Informática, desde el 2013 a la fecha, utilizando la plataforma de la carrera de Informática Empresarial, así como los cursos de Introducción a la Computación e Informática, Planificación Informática, Sistemas de Información impartidos desde que se implementó el uso de las aulas virtuales en la UCR, Moodle se ha convertido en una parte fundamental del quehacer universitario. Con esto Moodle se convirtió en un aliado y facilitador para la organización, la interacción con los estudiantes y los procesos de evaluación. 
En mi caso en particular, las opciones de evaluación que cotidianamente utilizo son foros, consultas, cuestionarios, glosario, wiki y tareas. Esta última en mayor cantidad ya que me parece muy útil para que los estudiantes puedan hacer entrega de sus tareas, exámenes, proyectos de investigación, entre otros.

A la hora de calificar los entregables de los estudiantes, mantengo un registro donde se pueden hacer comentarios, desglosar la evaluación y poner la nota obtenida. Así cada estudiante puede saber el resultado que obtuvo y las observaciones del docente. Además, esta misma información se les envía automáticamente vía correo electrónico al estudiante inmediatamente después de que se calificó, en lugar de esperar hasta la siguiente clase presencial.

Las facilidades de acceso a equipos de cómputo y de Internet que ofrece la UCR a sus docentes y estudiantes vienen a apoyar y enriquecer el uso de herramientas como Moodle como un complemento a las lecciones presenciales.

De acuerdo con conversaciones con los estudiantes sobre el uso de las aulas virtuales, estos han manifestado que la encuentran como una herramienta de fácil uso. Claro está, son estudiantes de informática. Sin embargo, hoy día los jóvenes universitarios en general están identificados con el uso de las tecnologías. Al igual que en mi caso, los demás docentes de la carrera en la Sede de Occidente han implementado el uso de la plataforma obteniendo los mismos resultados, con una curva de aprendizaje muy corta y que ha permitido obtener todos los beneficios de Moodle. Esto ha permitido que el estudiante se familiarice desde el inicio de sus estudios universitarios con la plataforma y la vea como una parte fundamental de todos los cursos de carrera y se acostumbren a su uso.

Algo que me ha cautivado como docente en el uso de Moodle ha sido la interacción que se logra con los estudiantes en actividades donde se comparte con los demás, como los foros o las wiki, logrando una mayor participación de todos comparado con la participación obtenida en clases presenciales. Además, los motiva a investigar información adicional a la ofrecida y compartirla con el resto del grupo.

Hay que rescatar el hecho de que el uso de aulas virtuales viene a facilitar a los estudiantes el envío de tareas, sin necesidad de incurrir en tiempo y gastos de impresión, e incluso sin necesidad de trasladarse físicamente al recinto universitario para cumplir con las entregas. Siempre y cuando cuenten con acceso a Internet el proceso es simple.

Por otro lado, la facilidad de comunicación entre los estudiantes y con el profesor hace que la información fluya de manera adecuada, y se puede apoyar con el envío de correos electrónicos desde la misma herramienta. un repositorio de las fechas, archivos, los comentarios y la evaluación. Lo que 
evita incurrir en riesgos de pérdida de entregables o calificaciones por parte del profesor, y le garantiza al estudiante que todo el material elaborado a lo largo del curso está almacenado en un lugar seguro en caso de que lo requiera.

Para la UCR el uso de Moodle también representa un ahorro en papelería y otros insumos de oficina, ya que se reduce o elimina el uso de fotocopias o impresiones de actividades como tareas, pruebas cortas, cuestionarios y demás. Como parte de los buenos resultados del uso de la plataforma en la carrera de Bachillerato en Informática Empresarial se trabaja ahora en la creación de una nueva opción académica de posgrado totalmente virtual, la Maestría en Informática Empresarial, la cual está siendo diseñada por docentes del bachillerato. Además, existe la representación de las otras sedes regionales donde se imparte y que utilizará este tipo de herramientas para sus diferentes cursos.

\section{Conclusiones}

Las opciones tecnológicas de hoy nos obligan a los docentes a darle una mayor importancia a las herramientas que vienen a colaborarnos en el proceso de enseñanza-aprendizaje, dejando de lado el curso tradicional para incursionar en nuevas modalidades a la hora de impartir lecciones. Según Rama (2011), los cursos virtuales, más allá de que se apoyan en una estructura curricular, determinados recursos instruccionales y estructuras de enseñanza, ponen más el acento en el autoaprendizaje. Por tanto, tienden a requerir un nuevo paradigma evaluativo centrados en las competencias y los aprendizajes efectivos a través de la introducción de pruebas de evaluación.

A nivel internacional se están desarrollando herramientas tecnológicas que apoyan los procesos educativos y facilitan el proceso de enseñanza-aprendizaje. La educación superior cada vez más está utilizando la opción de cursos en línea o bimodales, dándole al estudiantado distintas maneras de recibir lecciones; lo que además, permite un mayor acceso a la educación.

En Costa Rica, al igual que en otros países, el uso de Moodle para aulas virtuales ha tomado gran auge y se utiliza cada vez más a nivel superior. La UCR basa su mediación virtual en esta plataforma para todos los cursos y talleres que lo requieran. Ha demostrado ser una novedosa opción académica que brinda a sus usuarios las características necesarias para implementar un curso y realizar evaluaciones de aprendizajes.

Por su parte, el uso de Moodle como plataforma de aulas virtuales para la carrera de Informática Empresarial de la Sede de Occidente se da en todos los cursos de la misma. El personal docente ha implementado poco a poco las evaluaciones mediante esta herramienta.

Las opciones de evaluación del Moodle permiten al usuario evaluar aprendizajes desde distintas modalidades, dando una gama de posibilidades según sea la necesidad o el tipo de contenido. 
La calificación de las evaluaciones se realiza desde el aula virtual, donde el docente puede ajustarlas a la evaluación planteada en la carta al estudiante. Y además, la herramienta realiza los cálculos necesarios para brindar las notas y promedios finales del curso, que además pueden ser exportados a archivos en diferentes formatos.

El uso de Moodle para evaluar aprendizajes en la carrera se ha convertido en una práctica de evaluación emergente, que está siendo compartida a las demás sedes de la UCR.

\section{Referencias}

Alpízar, E. S. (2011). Resultados del primer curso de traducción completamente virtual e interactivo en Costa Rica. Revista de Lenguas Modernas.

Casales, Rojas y Paulí (2008) Casales, R.; Rojas, J. \& Paulí, G. (2008). Algunas experiencias didácticas en el entorno de la plataforma Moodle. Revista de Informática Educativa y Medios Audiovisuales, 5(19), 1-10.

Martínez de Lahidalga (2008) Ros Martínez de Lahidalga, I. (2008). Moodle, la plataforma para la enseñanza y organización escolar.

Molist (2008) Molist, M. (2008). Moodle llena la geografía educativa española de campus virtuales. Diario el Pais.

Rama (2011) Rama, C. (2011). Evaluación en la educación no presencial: Desde el paradigma tradicional de evaluar procesos de enseñanza (indicadores educativos) a modelos emergentes de evaluación del aprendizaje (competencias adquiridas). El aseguramiento de la calidad de la educación virtual. Perú: Editorial Gráfica Real, 25-42.

Reyes, Cabrera, Estévez, Jiménez y Limaya (2005) Reyes, R. A.; Cabrera, N. C., Estévez, O.; Jiménez, G.; Limaya, G. \& Barba, M. (2005). La evaluación del aprendizaje usando las actividades de Moodle. In 3rd International Conference on Multimedia and Information and Communication Technologies Education m-CTE2005.

Ros, I. (2008). Moodle, la plataforma para la enseñanza y organización escolar. Ikastorratza, e-Revista de Didáctica 2. Retrieved from http://www.ehu.es/ikastorratza/2_alea/ moodle.pdf

Silvio (2000), Silvio, J. (2000). La virtualización de la Universidad: ¿Cómo transformar la educación superior con la tecnología? 13. IESALC/UNESCO.

Moodle como herramienta de evaluación: la experiencia del Bachillerato de Informática Empresarial en la UCR Sede de 60 . Occidente 